\title{
Delay Tolerant Routing Protocol Modeling for Low Power Wearable Wireless Sensor Networks
}

\author{
Muhannad Quwaider \\ Computer Engineering \\ Jordan University of Science and Technology, Jordan \\ mqquwaider@just.edu.jo
}

Subir Biswas

Electrical and Computer Engineering

Michigan State University, USA

sbiswas@egr.msu.edu

Received: July 5, 2012 Accepted: September 5, 2012 Published: September 29, 2012

DOI: $10.5296 /$ npa.v4i3.2054

URL: http://dx.doi.org/10.5296/npa.v4i3. 2054

\begin{abstract}
A structural model for packet store-and-forward routing protocols in Wireless Body Area Networks (WBAN) is presented in this paper. WBAN has been experimentally constructed in order to illustrate and capture on-body topology disconnections. The discontinuity of the topology outcomes of the presence of ultra-short transmission range radio links, unexpected Radio Frequency (RF) attenuation, and the mobility of the human posture. Energy harvesting model for ultra-short transmission range on-body sensor is proposed. Then, these sensors are used for demonstrating delay techniques for evaluating on-body single-copy Delay Tolerant Network (DTN) packet routing protocols. In this paper, source-to-destination packet routing delay for different protocols like, opportunistic, random-based, utility-based and other protocols that capture multi-scale neighborhoods in human postural mobility has been constructed and evaluated. Then, performance results of the proposed protocols are evaluated experimentally and compared with the results obtained via the model that is developed. Through multi-scale modeling of the on-body spatio temporal neighborhood of the link disconnection patterns, it is shown that the proposed protocols can provide enhanced routing performance compared with a number of existing opportunistic, utility and random based DTN packet routing protocols.
\end{abstract}




\section{1) Macrothink}

Keywords: Wireless Body Area Networks, Low-power Sensor, Ultra-short Transmission Range Transceiver, Delay Tolerant Network, Packet Routing Protocol, Delay Modeling.

\section{Introduction}

In many recent applications, many of small wireless sensors are strategically implanted or placed on the body of the patient, can generate a network called Wireless Body Area Network or WBAN [1]. This WBAN can be used strictly for monitor human subject vital signs, then providing online and on current time feedback which enabling service provided for many patient diagnostics processes through continuous monitoring of chronic situations, recovery development from a disease or operating procedure. On-body data transmission across sensors can be node to node or multi-node to node. The distributed detection of player's posture [2] require node to node data exchange across different on-body nodes. Fig. 1shows applications such as monitoring vital signs that need all body-implanted or mounted nodes [3] to send data as multi-node to node and finally to a destination node, in which can rely and process the data information in a wireless way to an out-of-body server.

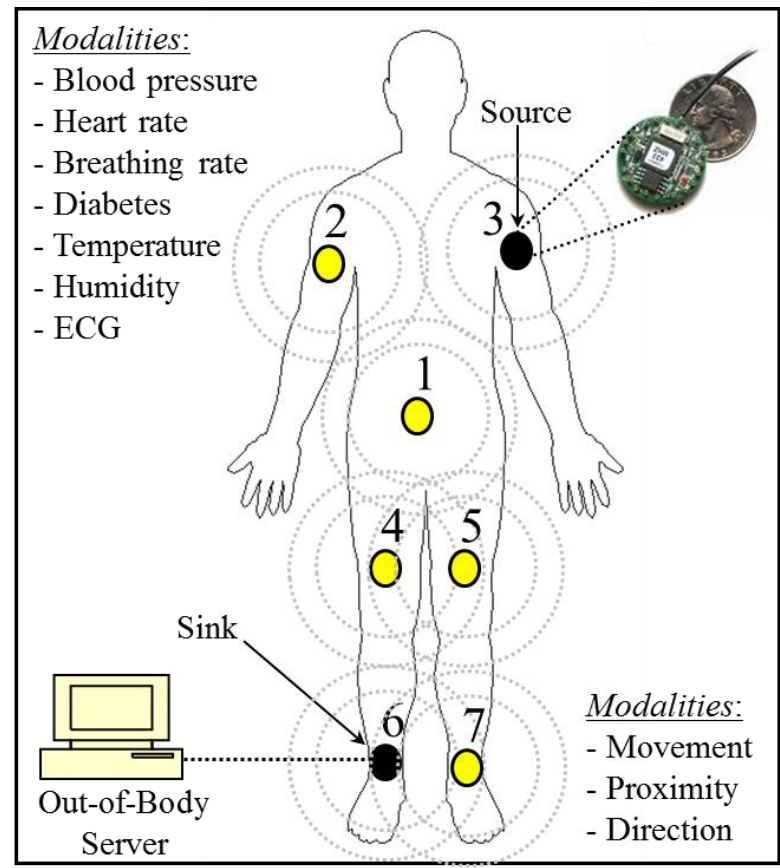

Figure 1, Wireless Body Area Network

Data transaction to and from on-body sensors can be also online or non-online. Although patient monitoring type of applications would require online packet routing, monitoring a player's physiological data can be collected offline for post-processing and analysis purposes. The routing protocols modeled in this paper provide to this non-online class of on-body applications. Therefore, on-body packet routing schemes are analyzed and modeled in this paper in a network with partitioning because of the postural body movement and short transmission range sensors. 
The low power sensor for embedded applications with limited energy [4], sometimes the energy is supplied by harvested processes [5], suffer also for low-power Radio Frequency (RF) transceivers and short transmission range restriction, and. This scenario application includes on-body implanted body sensors. In literature, short transceivers range can be with transmission ranges like from 0.0 to $1.0 \mathrm{~m}$ as in [4], from 0.2 to $1.0 \mathrm{~m}$ as in [5], from $0.2 \mathrm{~m}$ as in [6], and from 0.0 to 1.0 as in [7]. With required transmission powers changed between $0.75 \mathrm{~mW}$ to $6 \mathrm{~mW}$, which are within a range that can be handled with common harvesting techniques such as piezo-electric generation from body movements.

Low RF ranges also mean that body movements can give rise to frequent partitioning or disconnection in the topologies, which will cause Delay Tolerant Network (DTN) [8] in WBAN. This network apportioning can frequently become worse and worse by the unexpected RF attenuation due to signal obstruction by clothing and body segments. In real-time applications, such as patient monitoring may not be supported in the presence of topological partitioning, non-online applications such as player's physiology monitoring can still be supported using on-body DTN packet routing across disconnected partitions. Performance goals for such protocols will be to obtain low end-to-end delay, low packet loss, and low transmission energy consumption.

Our goal in this paper is to construct an energy-harvesting mode that can be used for developing packet transfer delay as analytical modeling algorithms for a set of DTN routing protocols which can be applied in human body topology. The majority of delay in DTN routing originates due to the packet storing in different partitions caused by topological disconnections. In this paper first, we propose an energy-harvesting model for on-body wearable sensor. Energy-harvesting sources vary widely in the energy available in BAN, but here we will focus on energy harvesting taking energy from the vibration of the piezo-electric sensor and accelerometer data measurement. Second, architecture of WBAN is developed to construct routing problem with on-body topology and leading to DTN routing protocols on-body routing experiments that are modeled and evaluated. Third, we develop a mechanism for collecting a topology trace for wirelessly extracting network topology, where the extracted topology is a function of human dynamics posture. At the end, analytical mechanisms are developed for demonstrating the packet end-to-end delay for different DTN routing protocols, like, opportunistic [9], [10] utility based [9], [11], random [9], [12] and PRMPL [13].

\section{Related Work}

The current WBAN schemes [1], [14], [15] assume tree and star graph with the constructed topologies, which having a source to sink existing path between any two on-body nodes at any time. Nevertheless, the proposed schemes do not consider DTN routing pattern to handles graphs with. In literature, routing in DTNs can be characterized [8], [16] like: 1)single copy [10], [11], and 2) Multiple copy [17], [18]. With multiple copy schemes a number of packet copies are circulated through many moveable nodes to increase the possibility of delivering the packet to the destination. On the other hand, the work proposed 
in [19-21] is based on the contacts history in order to create a neighborhood Index and then determine the most appropriated route for DTNs. However these schemes provide low packet delay, the main restriction with these schemes is the size overheads and the energy caused by extreme packet broadcasts. With energy limitations WBANs, these expenses are not applicable. By single copy packet routing schemes, information about the dynamic topology is used in order to reduce the delay without multiple replication. With single copy schemes, a node forward a packet to another node when the encountered node has more probability to encounter the destination that the current node.

To have these proposed schemes working as whatever outside viral/epidemic routing [22], the nodes should have assured degree of temporal and spatial neighborhood in their mobility and connecting forms. The scheme PROPHET [17], which is an extension of epidemic routing, develops a probabilistic framework for capturing the spatio-temporal neighborhood present in the node mobility pattern within a dynamically partitioned wireless network. The authors in [23] define a high-dimensional Euclidean space, called MobySpace, constructed upon nodes' mobility patterns. The specific MobySpace evaluated is based on the neighborhood of movements defined as the frequency of visits of nodes to each possible location.

Opportunistic [9], [10] and random-based [10] are also two DTN routing protocols that are studied for other applications, where no neighborhood information are used for evaluations. While a packet with opportunistic routing protocol is delivered directly from the source to destination, when the link is available, otherwise the source keeps buffering the packet. With random-based routing, a node forward a packet randomly like the logic in [24]. These schemes do not perform well because the lake information of the neighborhood.

The all above schemes are applied to networks spanning across local to wide areas, few extending all the way up to the inter-planetary scale [25]. The objective in our work is to apply the key disconnected routing concepts, as identified above, in an ultra-short transmission range body area environment. The key challenge is to develop mechanisms for capturing the locality of on-body node movements caused by human postural mobility, and to apply them for packet routing.. In this paper we will model the packet routing delay for very well-known DTN routing schemes. Then, we will compare between schemes that capture neighborhood in single-scale topology and with schemes that capture neighborhood in multi-scale topology with posture mobility. In our study we will include opportunistic [9], [10], [24], random-based [10], [24], utility-based [10], [11], [24] and PRMPL [13], which is precisely implemented for on-body DTN routing protocol.

\section{Experimental Characterization}

\subsection{WBAN Prototype}

A Wireless Body Area Network (WBAN) is constructed by mounting seven sensor nodes (attached on two upper-arms, two thighs, two ankles and one in the waist area) as shown in Fig. 1. Each wearable node consists of a 900MHz Mica2Dot MOTE (running TinyOS operating 
system), with Chipcon's SmartRF CC1000 radio chip (chipcon.com), and the sensor card MTS510 from Crossbow Inc. (www.xbow.com). The Mica2Dot nodes run from a 570mAH button cell with a total sensor weight of approximately 10 grams. The default CSMA MAC protocol is used with a data rate of $19.2 \mathrm{kbps}$.

Via software adjustments of the CC1000's transmission power, the transmission range is set to be in between $0.3 \mathrm{~m}-0.6 \mathrm{~m}$. By doing so, we are able to emulate the ultra-low transmission range for the embedded transceivers [4-7] as reported in the literature. Note that the variation of the range is caused due to the variability in antenna orientation, clothing, and other on-body RF attenuation characteristics.

All experiments in this paper correspond to multi-node to node routing in which data from all other nodes are sent to node-6 (see Fig. 1), which is designated as the on-body destination node. This node collects raw data, and sends processed results to an out-of-body server using a wireless link. This external link is created between the on-body destination and to an out-of-body Mica2Dot node connected to a PC through a custom-built serial interface, running RS232 protocol.

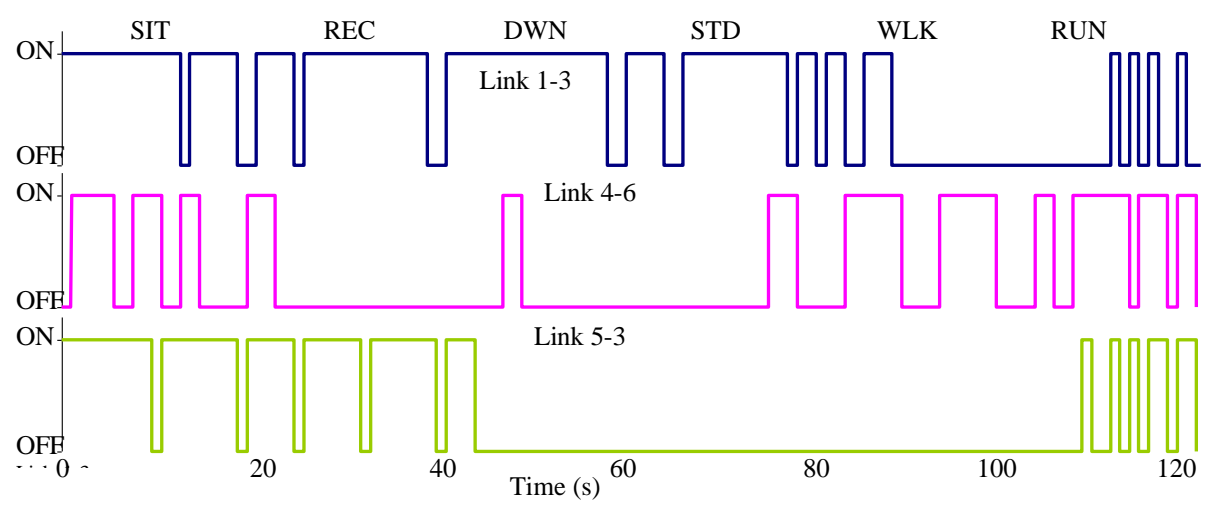

Figure 2, Link state with postural variation

\subsection{Network Partitions and Topology Variations}

A subject, fitted with seven sensors, was asked to follow a pre-determined posture sequence (SITTING, SIT-RECLINING, LYING-DOWN, STANDING, WALKING and RUNNING), each lasting for $20 \mathrm{sec}$. The status of three WBAN links (1-3, 4-6, and 5-3) during such an experiment is shown in Fig. 2.

As shown in Fig.2, during specific postures, like SIT and REC, some links, like 3-5 (the left arm and the left thigh) is connected most of the time. As noticed, these postures help to keep this link close. While with other postures, like STANDING, WALKING and LYING-DOWN, this link is frequently disconnected. Similar behaviors can be noticed with other links, like 1-3 (the chest and the left arm) and 4-6 (the right thigh and the right ankle). Therefore some links are connected merely within specific postures which making postural variations in on-body network topology. On the other hand, even with connected posture, it may few disconnections in the link due to signal obstruction by clothing or body segments 
and slightly body movement. Example of this behavior as shown in the figure, link 1-3 at time 0 to $20 \mathrm{sec}$ and during the SIT posture.

In order to model different DTN routing protocols, we develop a mechanism that collect the topology of the network from WBAN prototype in Fig. 1 and as processed in Fig. 2. Each node and during the human subject experiment, sends the neighborhood information to a PC-server out of body. The neighborhood information includes the state of the neighbor link $\mathrm{OFF} / \mathrm{ON}$ and the time stamp of the link state. Then, these topology trace information is used for modeling the all routing protocols that are proposed in this paper.

\subsection{Characterization of Energy Harvesting}

BANs applications require long lifetime, data accuracy, and energy efficiency. In general, the battery in a sensor node is limited and it is not easy to replace it, especially the implanted sensors. Since wireless communications consume most of the energy in typical WBAN, an energy efficient data communication scheme is greatly desired. Hence the network needs to ensure data routing in addition to ease of deployment, energy efficiency and low cost.

Energy-harvesting sources vary widely in the energy available in BAN, but here we will focus on energy harvesting taking energy from the vibration of the piezo-electric sensor and accelerometer data measurement. Thus, although increasing battery life through harvesting would revolutionize BANs, more explore is needed to create highly efficient hybrid solutions that incorporate energy generation, storage and usage for data routing as part of energy-aware routing protocol. Energy-aware routing should also work in addition to the network petitioning caused by ultra-low transmission power sensor and postural body movement.

The maximum harvesting power [26] using piezoelectric cell is given by:

$$
P=\frac{d s^{2} \cdot M^{2} \cdot \omega^{2} \cdot \alpha^{2}}{4 \cdot D^{2} \cdot C}
$$

where $M$ is damper mass, $\alpha$ is force factor, $D$ is damper factor, $\omega$ sampling frequency, $R$ is piezoelectric circuit resistor, $C$ is piezoelectric circuit capacitance, $a_{x}$, and $a_{y}$ are the accelerometer readings in $x$ and $y$ axes respectively, $d s$ is piezoelectric displacement and it is given by:

$$
d_{s}=\sqrt{X^{2}+Y^{2}}
$$

$X$ and $Y$ are the displacements in $x$ and $y$ axes respectively and they are computed as a function of the piezoelectric accelerometers readings, $\mathrm{mx}$ and my values in $x$ and $y$ axes, respectively [27].

During the time interval $\Delta t$ and according to Eqn. 1, the harvested energy can be computed as:

$$
E=P \cdot \Delta t
$$

In order to characterize the above model, the accelerometer data from node- 1 is collected during the experiment that was conducted in Section 3.2. Then the accelerometer data (mx 


\section{Macrothink}

and my) are used to calculate the piezoelectric sensor displacement, as shown in Eqn. 2 and according to [27], by which the harvested energy is computed. Fig. 3 shows these results. Same parameter settings reported in the literature [25], [26] are applied for computing the energy in Eqn. 3. As seen in the figure, the harvested energy is increased by increasing the sensor node displacement where that was during the WALK and RUN postures.

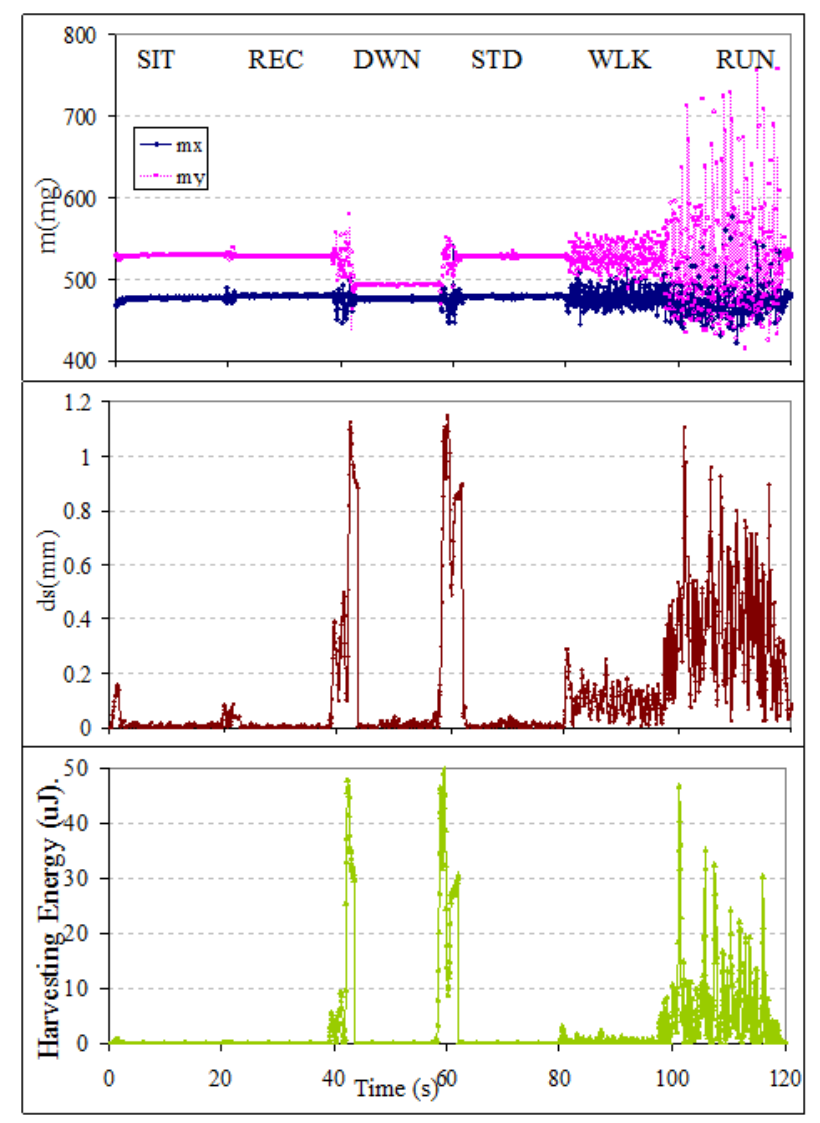

Figure 3, Waist sensor energy harvesting characterization

The second observation that can be made from Fig. 3 is that, with static postures, like SITTING, SIT-RECLINING, LYING-DOWN and STANDING, in which most of the people used to be, the node will be energy consumer. Therefore, a node with this model will be ultra-low power most of the time.

\section{Modeling DTN Routing Protocols}

The link state of $i$ and $j$ nodes at time slot $n^{\text {th }}$ can be defined by $L_{i, j}(n)$, which takes value of 0 for disconnected and 1 for connected. Then, the Link Probability Disconnection (LPD) of the defined link can be characterized as $\hat{P}_{i, j}(k)$, which represents the probability that $L_{i, j}(n)$ link stays disconnected for $k$ successive time slots after an arbitrarily chosen time slot. Through $T$ long time slots experiment trace topology with $n_{k}$ occurrences of $k$-slot of disconnections, the 
LPD can be sated by:

$$
\hat{P}_{i, j}(k)= \begin{cases}n_{k} / T, & \text { for } k \geq 1 \\ \sum_{n=1}^{T} L_{i, j}(n) / T, & \text { for } k=0\end{cases}
$$

The case $k \geq 1$ corresponds cases where randomly selected time slot is a part of one of $T_{\text {off }}$ periods or the last time slot during one of $T_{\text {on }}$ periods. Likewise, the case $k=0$ corresponds to situations where the randomly selected time slot is a part of one of the $T_{\text {on }}$ periods or the last time slot during one of the $T_{\text {off }}$ periods. From Eqn. 4 we will have $\sum_{k=0}^{T} \hat{P}_{i, j}(k)=1$, and the expected value of $\hat{P}_{i, j}(k)$ can be expressed as:

$$
E L D_{i, j}=\sum_{k=0}^{T} k \cdot \hat{P}_{i, j}(k)
$$

which represents the average number of disconnection time slots after a randomly chosen time slot. $E L D_{i, j}$ in Eqn. 5 can be also defined as $T_{o f f}^{i, j} / 2$, where $T_{o f f}^{i, j}$ is the average disconnection time slots duration.

\subsection{Modeling Opportunistic Routing Protocol (OPPT)}

With OPPT routing [9], [10], [24] a packet will be buffered in the source node until a direct link connection between the source and the destination. Even though, OPPT routing protocol is considered the simplest DTN routing scheme, the delay of this approach is very large compare with other approaches, especially with rarely link connections between the source and destination with low mobility network. Therefore, a data packet is delivered from the source $s$ to destination $d$ when $L_{s, d}=1$, and $E L D_{s, d}$ value computed in Eqn. 5 represents the delivery delay of the packet. The all generated packet during $T_{\text {off }}$ period can be delivered from the source to destination without additional delay if the rate of the generation packet is low enough during $T_{\text {off }}$ period, where the all packet be able to be delivered at the beginning of $T_{\text {on }}$.

On the other hand, when the rate of generating the packet is high during the $T_{\text {off }}$ period, an additional delay to $E L D_{s, d}$ of Eqn. 5 will be added due to the backlog clearance during the $T_{o n}$ period Throughout the $T_{\text {off }}$ period, assume the number of generated packets is $B$ with a rate of $\lambda$ and $B=\lambda \cdot T_{\text {off }}$. At the beginning of $T_{\text {on }}$ period the source node starts delivering these $B$ packets to the destination. The B packets need B time slots to be delivered because each delivered in one time slot. With the same packet generating rate, and during B slot, there will be another $B . \lambda$ packets will be generated. Then, these $B . \lambda$ packets need $B . \lambda$ another time slots to be delivered to the destination.

By merging ELD delay with the backlog clearance delay, the average packets delay of the created packets in $T_{\text {off }}$ interval is expressed by $E L D_{s d}+\sum_{i=1}^{B-1} i / B$. While the average packets delay 
created in the $T_{o n}$ period is expressed by $\frac{B}{2}+\sum_{i=1}^{B \lambda-1} i$. So, the overall opportunistic routing average packet delay is written as:

$$
D_{\text {OPPT }}=\frac{B \cdot\left(E L D_{s d}+\sum_{i=1}^{B-1} i\right)+B \cdot \lambda \cdot\left(\frac{B}{2}+\sum_{i=1}^{B \lambda-1} i\right)}{B+B \cdot \lambda},
$$

where $B=\lambda \cdot T_{o f f}^{s, d}=2 \cdot \lambda \cdot E L D_{s, d}$, and ELD is computed from Eqn. 5 .

\subsection{Random-based Routing (RAND)}

A node with a data packet in random-based routing protocol forward the data packet to a random neighbor if it does not have a direct link to the destination as also define in [10], [24]. Afterward, this packet is forwarded randomly till a node has a direct connection with the destination.

With RAND routing, a node with a packet, it sends that packet randomly consistent to any of it's neighbors. Hence, the probability of forwarding a packet from node $i$ to node $j$ and at time slot $n$ can be expressed by:

$$
P_{i, j}^{f}(n)=\frac{L_{i, j}(n)}{\sum_{j=1}^{N} L_{i, j}(n)}, \text { for all } j \in N, j \neq i, j \neq d
$$

where $N$ is the number of nodes in the network. Eqn. 7 is applicable as long as node $i$ currently has at least one neighbor, or $\left(\sum_{j=1}^{N} L_{i, j}(n) \neq 0\right)$ and $j \neq d$, where there is no connection with the destination $\left(\right.$ i.e. $\left.L_{i, d}(n)=0\right)$. When node $i$ does not have neighbors, it keeps buffering the packet with $\left(P_{i, i}^{f}(n)=1\right)$ and $P_{i, j}^{f}(n)=0$, for all $j \neq i$. While, when node $i$ has a connection with the destination $d$ as one neighbor, node $i$ directly forwards the packet with probability of 1 to destination $d$.

Let us define forwarding matrix $A(n)$ at time slot $n$ as a matrix which defines the probabilities of forwarding packets through the all likely neighbors of $N$ nodes network as follows:

$$
A(n)=\left[\begin{array}{ccccccc} 
& 1 & 2 & \cdots & j & \cdots & N \\
1: & P_{1,1}^{f}(n) & P_{1,2}^{f}(n) & \cdots & P_{1, j}^{f}(n) & \cdots & P_{1, N}^{f}(n) \\
2: & P_{2,1}^{f}(n) & P_{2,2}^{f}(n) & \cdots & P_{2, j}^{f}(n) & \cdots & P_{2, N}^{f}(n) \\
: & : & : & \cdots & : & \cdots & : \\
i: & P_{i, 1}^{f}(n) & P_{i, 2}^{f}(n) & \cdots & P_{i, j}^{f}(n) & \cdots & P_{i, N}^{f}(n) \\
: & : & : & \cdots & : & \cdots & : \\
d: & 0 & 0 & \cdots & 0 & \cdots & 0 \\
: & : & : & \cdots & : & \cdots & : \\
N: & P_{N, 1}^{f}(n) & P_{N, 2}^{f}(n) & \cdots & P_{N, j}^{f}(n) & \cdots & P_{N, N}^{f}(n)
\end{array}\right]
$$


The Forwarding Matrix is constructed using Eqn. 7 and the following notable comments can be made from $A(n)$. (1) The all $d^{\text {th }}$ row values are zeros because a packet is delivered at the destination $d$ and no more forwarding is needed. (2) The $d^{\text {th }}$ column values are either zero or 1 , because node $d$ either connected or not connected with a node as shown in Eqn. 7. (3) The addition of the all values in every row (except the $d^{\text {th }}$ row) is equal to 1 .

To construct the delivery delay of RAND protocol, let us study the source node $s$ generate a packet at time slot $n$, and that is delivered at time slot $n+k$ to destination $d$, with packet delay of $k$ slots. Let us define $\rho_{s, d}^{n}(k)$ to be the probability of delivering the packet from $s$ to $d$ with a delay of $k$ as follows:

$$
\rho_{s, d}^{n}(k)=[A(n) . A(n+1) \ldots \ldots . A(n+k)]_{s, d}=\left[\prod_{i=0}^{k} A(n+i)\right]_{s, d}
$$

$\rho_{s, d}^{n}(k)$ is the $[\mathrm{s}, \mathrm{d}]$ element from $A(n)$ forwarding matrix that is define in Eqn. 8. Then, to compute the expected delay of RAND protocol or $D_{R A N D}$, the value of Eqn. 9 is multiplied by $k$ slots for all possible $k$ values for a packet generated at time slot $n$ or as follows:

$$
D_{R A N E} \sum_{k=0}^{T} k \cdot \rho_{s, d}^{n}(k)=\sum_{k=0}^{T} k \cdot\left[\prod_{i=0}^{k} A(n+i)\right]_{s, d}
$$

where $T$ is the experiment length which can be considered the maximum value of $k$.

\subsection{Link Neighborhood for Utility-based Routing}

With random-based forwarding, a node forwards a packet randomly without considering the neighborhood connectivity with other nodes. While with utility-based forwarding protocols (also reported in [9-11], [17], [24]) a node forwards a packet to a neighbor with newest meeting with the destination. Therefore, the utility-based routing protocol leverages the age of the link during the packet forwarding. With utility-based routing a utility value is computed at each node, where it is maximized when a node encounters the destination and this value reduced every time a node does not encounter the destination. A node forwards a packet to a neighbor with the highest utility value.

Let us define $U_{i, j}(n)$ be the utility value at time slot $n$ and at node $i$ against node $j . U_{i, j}(n)$ is maximized when node $i$ encounters node $j$ and it is reduced by a certain value every time slot node $i$ and $j$ are not connected and as a function of time[10], [11]. The $U_{i, j}(n)$ can be expressed as:

$$
U_{i, j}(n+1)= \begin{cases}U_{\text {max }}, & \text { if } L_{i, j}(n+1)=1 \\ U_{i, j}(n)-1, & \text { if } L_{i, j}(n+1)=0\end{cases}
$$

here $U_{\max }$ is the maximum value. The information of the utility values is exchanged between 
the all neighbor nodes using Hello packets.

Using utility-based protocol, a packet at node $i$ is forwarded at time slot $n$ to node $j$ if $U_{i, d}(n)<U_{j, d}(n)$ and $U_{j, d}(n) \geq U_{k, d}(n), \forall k \in \psi_{i}(n)$, for all neighbors $\psi_{i}(n)$ for node $i$ at time slot $n$.

As in RAND routing protocol, the delay of the packet in UTILITY is computed using the same probability forwarding $P_{i, j}^{f}(n)$ in Eqn. 7, but with updated the formula of the formula as in UTILITY. In this case the forwarding probability is formulated as:

$$
P_{i, j}^{f}(n)=1, \text { if } U_{i, d}(n)<U_{j, d}(n), \forall i, j \in N
$$

where $N$ is the all nodes in the network. In this equation, if node $j$ has higher utility to destination than node $i$ at time slot $n$, then the forwarding probability $P_{i, j}^{f}(n)$ will be 1 , otherwise, node $i$ will keep buffering the data packet. Then, the forwarding matrix $A(n)$ constructed using the forwarding probabilities that are computed using Eqn. 12. Once the delivery probabilities $\rho_{s, d}^{n}(k)$ is computed using matrix $A(n)$, the UTILITY packet routing delay is calculated using Eqn. 10 as $D_{\text {UTLTYY }}=\sum_{k=0}^{T} k \cdot\left[\prod_{i=0}^{k} A(n+i)\right]_{s, d}$.

\subsection{Probabilistic Routing with Multi-scale Postural Locality (PRMPL)}

In this proposed PRMPL, we employed the Postural Link Cost (PLC) that is proposed in [13] which utilized the neighborhoods of the links of on-body topology in multiple scales of time. Same as in UTILITY routing, with PRMPL the forwarding probability is replaced by the PLCs derived in [13]. The utility value of PLC is adjusted by itself according to the human activity to maximize the utility of the next hop to destination. PLC can be expressed by $\beta_{i, j}(n), \quad\left(0 \leq \beta_{i, j}(n) \leq 1\right)$, by which, $L_{i, j}(n)=1$ is defined by computing the probability $\beta_{i, j}(n)$. $\beta_{i, j}(n)$ is updating according to the following expression [13]:

$$
\begin{array}{ll}
\beta_{i, j}(n)=\beta_{i, j}(n)+\left(1-\beta_{i, j}(n-1)\right) \cdot \omega & \text { if link } L_{i, j}(n)=1 \\
\beta_{i, j}(n)=\beta_{i, j}(n-1) \cdot \omega & \text { if link } L_{i, j}(n)=0
\end{array}
$$

where the constant $\omega(0 \leq \omega \leq 1)$ determines the rate of increasing and decreasing the cost of PLC or $\beta_{i, j}(n)$ of the link $L_{i, j}$ to the maximum value, which is 1 , or to the minimum value which is zero

With time invariant $\omega$, the PLC update rules in Eqn. 13 captures the neighborhood in short-term link connectivity in a manner conceptually similar to the age based utility 
formulation, as developed in [10], [11]. It is, however, not the same because in the designs in [10], [11], the routing utility of a link is increased incrementally when the link is formed, and is reduced to zero as soon as the link is disconnected. This formulation of utility misses out the fact that even after disconnection, the formation probability of that link may be higher than a currently-connected link. In other words, those definitions of utility fairly differentiate across currently connected links, but not across the currently non-connected links. In the formulation of PLC in Eqn. 13, motivated by the logic used in PROPHET [17], we track the short-term neighborhood even when a link is not physically connected. This extended persistency in PLC is expected to improve performance over the existing age based utility definitions as used in [10], [11].

According to the design in Eqn. 13, with large value of $\omega, \beta_{i, j}(n)$ increases fast and decreases slowly when the link connected or disconnected, respectively, which means a good historical link. While, with small value of $\omega, \beta_{i, j}(n)$ increases slowly and decreases fast when the link connected or disconnected, respectively, which means a bad historical link. From these observations, let us define HCQ to be the Historical Connectivity Quality at time slot $n$ of link $L_{i, j}$ in WBAN as:

$$
\omega_{i, j}(n)=\sum_{r=n-T_{\text {window }}}^{n} L_{i, j}(r) / T_{\text {window }}
$$

$T_{\text {window }}$ is the window size in time slot where the quality of the connectivity quality is computed. The constant $\omega_{i, j}(n),\left(0 \leq \omega_{i, j}(n) \leq 1\right)$ represents the link quality as a historic portion of time in last $T_{\text {window }}$ slots that the link was connected. We found experimentally $T_{\text {window }}$ to be in range of 7 to $14 \mathrm{sec}$.

Fig. 4 shows the performance of HCQ $\omega_{i, j}(n)$ and PLC $\beta_{i, j}(n)$ as a function of time. The first part of the figure shows the link activity $L_{i j}(n)$ and the performance of $\omega_{i, j}(n)$ as demonstrating in Eqn. 14 as a function of $T_{\text {window }}$, where $T_{\text {window }}$ is set to 7 slots. The second part of the figure shows the corresponding performance of $\beta_{i, j}(n)$ with $\omega$ values of 0.9 and 0.1. and the averaged computed value of $\omega_{i, j}(n)$ in Eqn. As shown in the figure, with good connected link, as in the first half of the graph, $\beta_{i, j}(n)$ decreases slowly, because of the large value of $\omega$ which reacts fine to a temporary disconnection and recovering rapidly 
when the link is reconnected again. On the other hand, with small $\omega, \beta_{i, j}(n)$ reacts unwell, as with $\omega=0.1$.

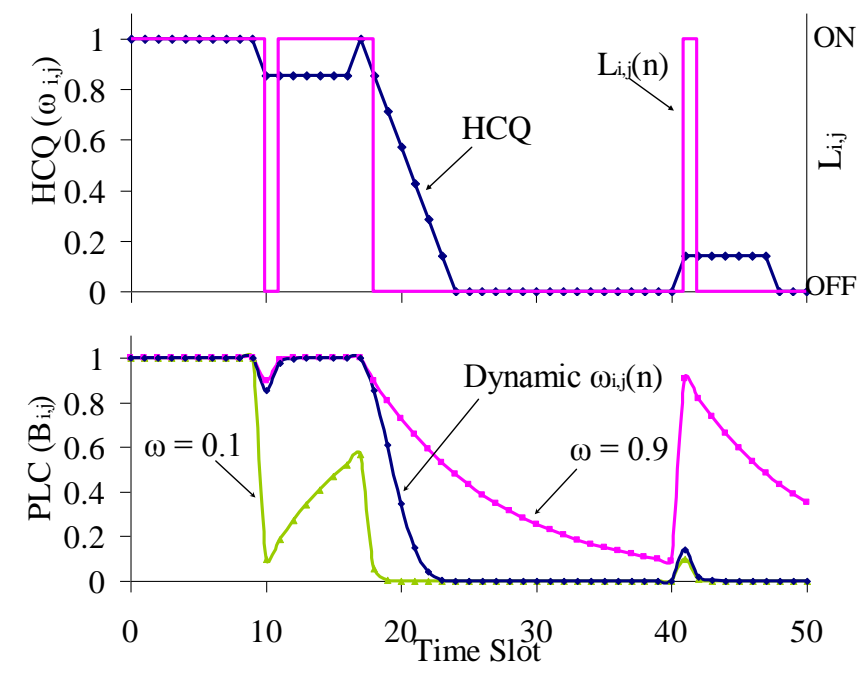

Figure 4, Evolution of multi-scale neighborhood in terms of PLC and HCQ

Same interpretations can be conducted for the second part of Fig. 4. In this case and with small $\omega$ value, $\beta_{i, j}(n)$ reacts well for a temporary connection, and vice versa as discussed above.

On the other hand, with dynamic $\omega, \beta_{i, j}(n)$ was able to handle the both cases of good link (with $\omega=0.9$ ) and bad link (with $\omega=0.1$ ). As seen, these conclusions show how the HCQ and PLC can be used for implementing utility-based routing protocol to handle the neighborhoods in short and long neighborhoods terms for dynamic on-body links.

To model PRMPL packet routing delay, same forwarding process of UTILITY routing delay can be used. But with the $U_{i, j}(n)$ of the utility function is replaced by $\beta_{i, j}(n)$ which is the link cost of the posture. Therefore, $A(n)$ (the forwarding matrix), $P_{i, j}^{f}(n)$ (the forwarding probabilities), and $\rho_{s, d}^{n}(k)$ ( the delivery probabilities) are computed using Eqns. 8, 9 and 13 respectively. Then, the packet delay is computed as $D_{P R M P L}=\sum_{k=0}^{T} k\left[\prod_{i=0}^{k} A(n+i)\right]_{s, d}$ as in Eqn. 9.

\section{Performance Results}

To evaluate the all routing protocols that are proposed in this paper, same WBAN prototype that is shown in Fig. 1 is used where seven sensor-nodes were used.. Data packets are generated from the source (node-3, the left arm) to the destination (node-6, the right ankle), 
which corresponds the longest packet routing scenario in WBAN. The model analysis results correspond to the analytical analysis of the collected topology trace data for the same actual conducted experiments. The forwarding probability matrix in Eqn. 8, is then computed using the collected topology trace data for the purpose of computing different routing delays.

\subsection{Channel Access Control}

A high level polling admission control is implemented to get rid of the collision due to CSMA MAC in Mic2Dot's TinyOS networking, where one node is assigned to poll the all other nodes in the network in a round-robin manner. Consequently, a node will not send the Hello and the data packets through the network until it is polled by the polling node. Each node is assigned $200 \mathrm{msec}$ to access the network with frame duration of $1.4 \mathrm{sec}$, which consists of seven slots in the network, one slot for each node. In order to have a node with ultra-short transmission rage, we adjust by software the transmission power of a node to have transmission range of $0.3 \mathrm{~m}-0.6 \mathrm{~m}$, while the polling node is assigned to transmit the polling packet by full power to make sure that all nodes receive this packet. Fig. 5 shows the polling mechanism that we used, where node time slot is divided into three $60 \mathrm{msec}$ sub-slot each and $20 \mathrm{msec}$ protector time between adjacent slots. The first sub-slot is used to send the polling packet with full power from the polling node, and the second sub-slot is assigned with low power for Hello and data packet transmitted between the nodes in the network.

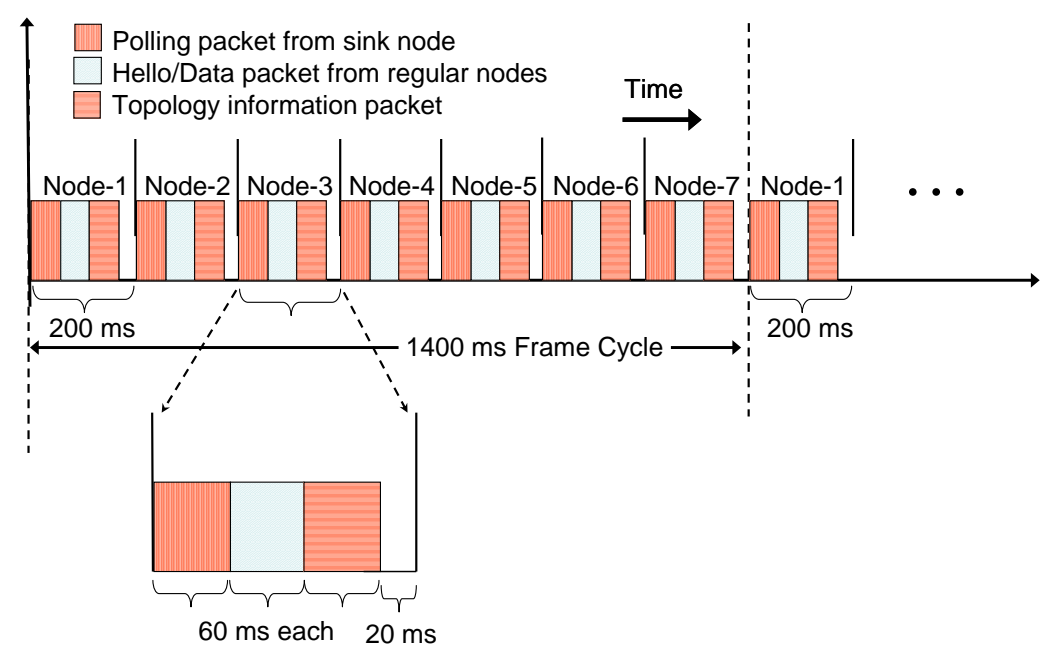

Figure 5, MAC Collision-free access via polling

While the third sub-slot is assigned with full power to send the topology trace data (neighbors information with time stamp) from each node to the polling node and then to out-of-body server, as seen in Fig. 1, and then to use these data in the model analysis.

\subsection{Performance Metrics and Traffic Generation}

Source to destination Packet Delay (PD) is the main performance metric that we are looking for and modeled in this paper, which is tried to be reduced by proposing the UTILITY and 
PRMPL based protocols. Other performance metrics, like Packet Hop Count (PHC) and Packet Delivery Ratio (PDR) are also reported to study the impact of improving on delay on these important parameters. Node- 3 is chosen to be the source and node- 6 is to be the destination in order to have the longest hop-to-hop routing. The source node generates packets with each packet size of 46 bytes every 4 time slots, where each time slot corresponds to $1.4 \mathrm{sec}$. All nodes are synchronized by the polling node which is node- 1 by sending the polling packet to all nodes with full power, as reported before in this section.

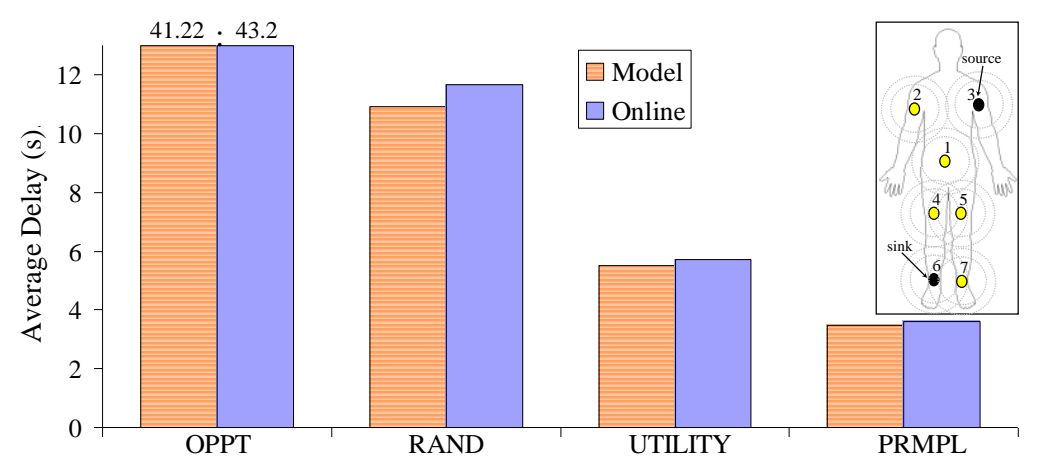

Figure 6, On-body packet delivery delay for different DTN routing protocols

\subsection{Packet Delay $(P D)$}

Source to destination packet delays from node- 3 to node- 6 is computed in this section for all proposed protocols reported in Section 4. Fig. 6 shows the delay results, where, a distinct experiment was conducted for each protocol. Each experiment was run for 22 minutes or $1320 \mathrm{sec}$. by sending 230 packets from the source to the destination. During each experiment, the human subject is asked to repeat 6 postures of SITTING, SIT-RECLINING, LYING-DOWN, STANDING, WALKING and RUNNING, where each posture lasts for 20 sec. Fig. 6 demonstrates the average packets delay calculated from the on-body experiment and the analytical model.

As seen in Fig. 6, the model-generated analytical and the experimental results are strictly equal through the all protocols. Other observation that we can make, UTILITY and PRMPL routing protocols perform lower delay compared with other routing protocols, because of using information about the topological neighborhood. However, PRMPL performs significantly lower delay compared with UTILITY and other routing protocols delay, because of using multi-scale topological neighborhoods information in human postural mobility by using $\beta_{i, j}(n)$ parameter, as described in Sections 4.4. RAND and OPPT both do not perform

well delay, because they do not make of use any topological neighborhood information with packet forwarding. Random-based routing shows slightly lower delay compared with OPPT, because WBAN is small network, and there are not that many possible packet forwarding. Therefore, it shows faster delivery compared with OPPT routing protocol. 


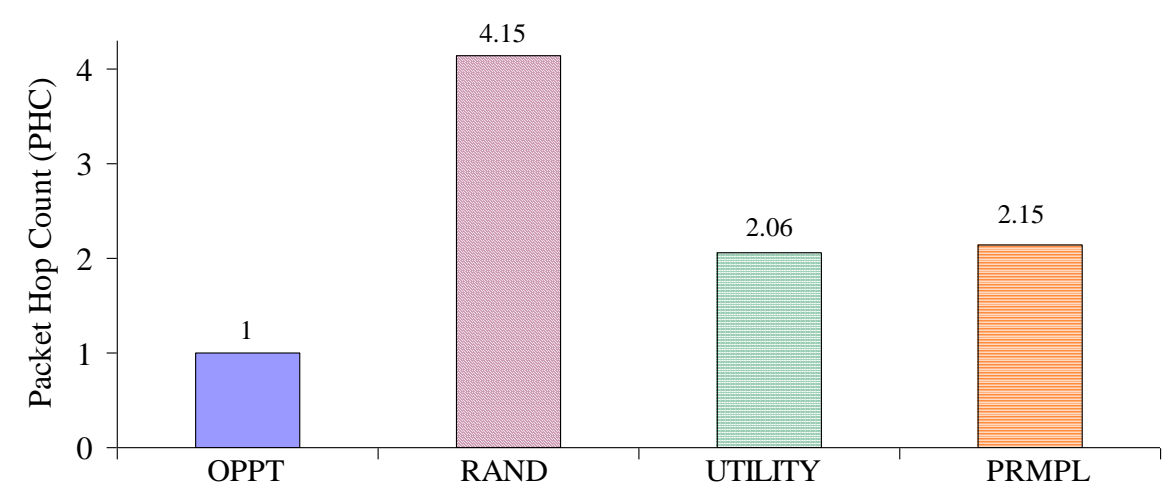

Figure 7, Average Packet Hop Count

\subsection{Packet Hop Count (PHC)}

PHC measurement helps to report how the protocol performs in term of on-body sensors transmission and reception energy spending. Fig. 7 shows the average hop count from the source to the destination. As shown in the figure, the RAND protocol needs a large number of PHC compared with other routing protocol, because of the randomized forwarding of the packet in this protocol. While, the OPPT protocol shows the lowest PHC value, which is 1 , because OPPT needs to have a direct contact between the source and destination to deliver the packet.

\subsection{Packet Delivery Ratio(PDR)}

With the WBAN prototype that we have, there is no link layer to retransmit the packet when it drops. Therefore, PDR is one important parameter need to be considered in packet forwarding in WBAN. The main reason for packet drops is due to the mobility during different human postures, where a neighbor may appear to be connected to a node at the time when a node has a packet needs to be transmitted, but actually it is no longer connected. This period is called blackout periods and it was chosen to be $2.8 \mathrm{sec}$. or two time frames. So, all proposed protocols in this paper suffer from these packets drops.

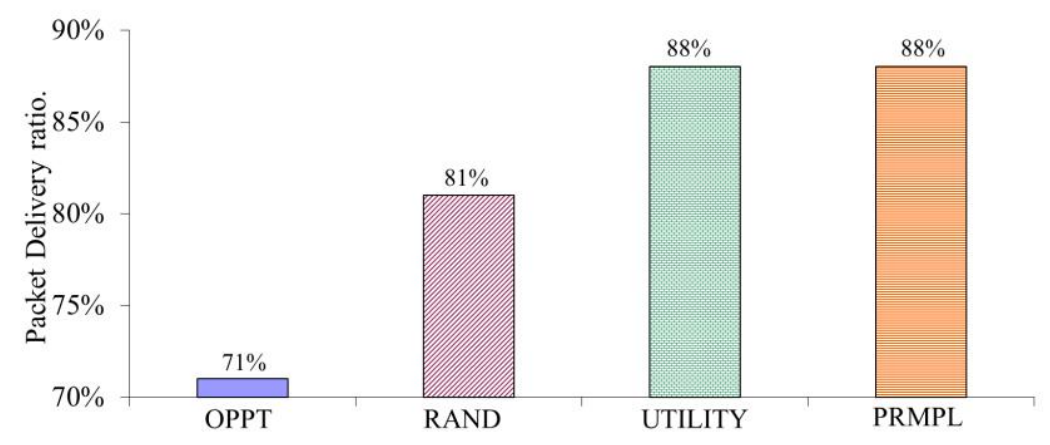

Figure 8, Packet delivery ratio for different protocols

Fig. 8 shows PDR performance results of the four routing protocols. Due to the sensitivity 
of the link between node-3 and node-6 (the source and the destination), the OPPT protocol shows very low PDR compared with other protocols. However, the large number of packet drops in RAND protocol is caused due to the large number of hop count or PHC in this protocol and as shown in Fig. 7. With large PHC, it causes to have more likely for a packet to be dropped due to the transmission during the blackout period. Therefore, there will be low PDR with RAND protocol compared with UTILITY and PRMPL. On the other hand, the UTILITY and the PRMPL protocols perform better in terms of PDR due to lower PHC of these two protocols, which suffer fewer packet drops due to the transient blackouts.

\subsection{Impacts of Postural Stability}

In the above experiments, the each posture lasts for $20 \mathrm{sec}$. The goal of this section is to study the impacts of the posture duration on the routing delay performance. The human subject is asked to repeat the same sequence of different postures of SITTING, SIT-RECLINING, LYING-DOWN, STANDING, WALKING and RUNNING, but with posture duration varying from 10 to $40 \mathrm{sec}$.

Fig. 9 summarizes the average packet delay of the four protocols as a function of posture duration. As shown in the figure, the OPPT delay performance is plotted with separate axis (to the right) in the same figure due to the significant high delay values of this protocol. The first observation that we can make from this figure is that the packet delays for all the protocols normally increase with higher posture durations. This is because with longer posture duration will impact to buffer the data packet for longer duration compared with shorter posture duration. The second observation that we can make on Fig. 9 is that relatively the all experimented and modeled protocols preserve the same performance behavior for packet delay as shown in Fig. 6.

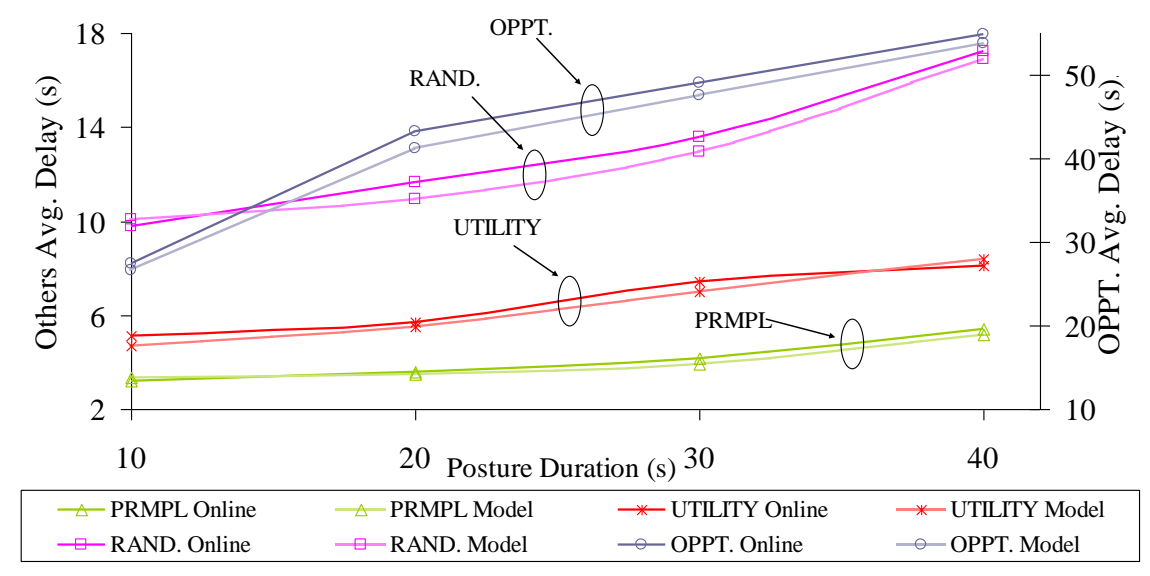

Figure 9, Packet delay variation with posture duration

\section{Conclusion and Future Work}

A delay modeling structure for Delay Tolerant Network (DTN) packet routing in 
Wireless Body Area Networks (WBAN) is developed in this paper. Using a system of WBAN for experimentally characterizing and capturing on-body topology traces, an analytical delay modeling technique was developed for evaluating single-copy DTN routing protocols. To emulate ultra-low power sensor, an energy harvesting model for ultra-short transmission range on-body sensor is proposed. Then, End-to-end routing delay for a set of protocols including opportunistic, random-based, utility based and other mechanism that capture multi-scale topological neighborhoods in human postural movements have been evaluated. Performance was evaluated experimentally and using the developed models. It was shown that via multi-scale modeling of the spatio-temporal neighborhood of on-body link disconnection patterns, it is possible to attain better delay performance compared to opportunistic, random-based and utility based DTN routing protocols in the literature. As ongoing work on this topic, we are going to develop an energy-aware routing protocol using the energy harvesting model that is proposed in this paper.

\section{References}

[1] E. Jovanov, A. Milenkovic, C. Otto, and P. C. de Groen, "A Wireless Body Area Network of Intelligent Motion Sensors for Computer Assisted Physical Rehabilitation," Journal NeuroEng. and Rehab, vol. 2, no. 11, p. 6, Mar. 2005, http://dx.doi.org/10.1109/IEMBS.2009.5334774

[2] K. Chen and D. J. Bassett, "The Technology of Accelerometry-based Activity Monitors: Current and Future," Med Sci Sports Exerc, vol. 37, pp. 490-500, 2005, http://dx.doi.org/10.1109/TSMCA.2004.836782

[3] M. Quwaider and S. Biswas, "Physical Context Detection using Multi-modal Sensing using Wearable Wireless Networks," Journal of Communication Software and Systems (JCOMSS'08), Special Issue on Medical Applications for WSN, vol. 4, pp. 191-202, 2008, http://dx.doi.org/10.1109/NCA.2010.36

[4] D. Sagan, "RF Integrated Circuits for Medical Applications: Meeting the Challenge of Ultra Low Power Communication," Ultra-Low-Power Communications Division, Zarlink Semiconductor, 2005, http://dx.doi.org/10.1109/ICSENS.2010.5690645

[5] S. Mikami, T. Matsuno, M. Miyama, M. Yoshimoto, and H. Ono, "A Wireless-Interface SoC Powered by Energy Harvesting for Short-range Data Communication," in Asian Solid-State Circuits Conference, 2005, 2005, pp. 241-244, http://dx.doi.org/10.1109/ASSCC.2005.251710

[6] E. Strömmer, M. Hillukkala, and A. Ylisaukkooja, "Ultra-low Power Sensors with Near Field Communication for Mobile Applications," International Conference on Wireless Network, ICWN'07, pp. 1-12, 2007, http://dx.doi.org/10.1109/SENSORCOMM.2008.96

[7] T. Falck, H. Baldus, J. Espina, and K. Klabunde, "Plug 'n Play Simplicity for Wireless Medical Body Sensors," in Pervasive Health Conference and Workshops, 2006, 2006, pp. 1-5, http://dx.doi.org/10.1109/PCTHEALTH.2006.361702

[8] Evan P.C. Jones and Paul A.S. Ward, "Routing strategies for delay-tolerant networks," ACM Computer Communication $\quad(C C R), 2006 . \quad$ Disponible en: http://citeseerx.ist.psu.edu/viewdoc/download?doi=10.1.1.119.6510\&rep=rep1\&type=pdf

[9] T. Spyropoulos, K. Psounis, and C. S. Raghavendra, "Efficient routing in intermittently connected mobile networks: The multiple-copy case," IEEE/ACM Transactions on Networking (TON), vol. 16, 
no. 1, pp. 77-90, 2008, http://dx.doi.org/10.1109/TNET.2007.897964

[10] T. Spyropoulos, K. Psounis, and C. S. Raghavendra, "Efficient Routing in Intermittently Connected Mobile Networks: The Single-Copy Case," Networking, IEEE/ACM Transactions on, vol. 16, no. 1, pp. 63-76, 2008, http://dx.doi.org/10.1109/TNET.2007.897962

[11] H. Dubois-ferriere, M. Grossglauser, and M. Vetterli, "Age matters: Efficient route discovery in mobile ad hoc networks using encounter ages," 2003. [Online]. Available: http://eprints.kfupm.edu.sa/23199/. [Accessed: 02-Dec-2009].

[12] M. Shin, S. Hong, and I. Rhee, "DTN routing strategies using optimal search patterns," in Proceedings of the third ACM workshop on Challenged networks, San Francisco, California, USA, 2008, pp. 27-32. http://dx.doi.org/10.1145/1409985.1409992

[13] M. Quwaider and S. Biswas, "DTN Routing in Body Sensor Networks with Dynamic Postural Partitioning," Elsevier Ad Hoc Networks, Volume 8, Issue 8, November, 2010. http://dx.doi.org/10.1016/j.adhoc.2010.03.002

[14] B. Braem, B. Latre, I. Moerman, C. Blondia, and P. Demeester, "The Wireless Autonomous Spanning tree Protocol for Multihop Wireless Body Area Networks," in Mobile and Ubiquitous Systems - Workshops, 2006. 3rd Annual International Conference on, 2006, pp. 1-8, http://dx.doi.org/10.1109/MOBIQW.2006.361753

[15] B. Latre, B. Braem, I. Moerman, C. Blondia, E. Reusens, W. Joseph, and P. Demeester, "A Low-delay Protocol for Multihop Wireless Body Area Networks," in Mobile and Ubiquitous Systems: Networking \& Services, 2007. MobiQuitous 2007. Fourth Annual International Conference on, 2007, pp. 1-8, http://dx.doi.org/10.1109/MOBIQ.2007.4451060

[16] P. Tsao and S. Nguyen, "BPTAP: A new approach toward IP over DTN," in 2012 IEEE Aerospace Conference, 2012, pp. 1 -5, http://dx.doi.org/10.1109/AERO.2012.6187106

[17] A. Lindgren, A. Doria, and O. Schelén, "Probabilistic routing in intermittently connected networks," SIGMOBILE Mob. Comput. Commun. Rev., vol. 7, no. 3, pp. 19-27, 2003, http://dx.doi.org/10.1109/INFCOM.2007.201

[18] E. Altman and F. De Pellegrini, "Forward correction and fountain codes in delay-tolerant networks," IEEE/ACM Trans. Netw., vol. 19, no. 1, pp. 1-13, Feb. 2011, http://dx.doi.org/10.1109/INFCOM.2009.5062111

[19] E. C. R. de Oliveira and C. V. N. de Albuquerque, "NECTAR: a DTN routing protocol based on neighborhood contact history," in Proceedings of the 2009 ACM symposium on Applied Computing, 2009, pp. 40-46.

[20] V. N. G. J. Soares, F. Farahmand, and J. Rodrigues, "A layered architecture for Vehicular Delay-Tolerant Networks," in IEEE Symposium on Computers and Communications, 2009. ISCC 2009, 2009, pp. 122 -127, http://dx.doi.org/10.1109/ISCC.2009.5202332

[21] V. N. G. J. Soares, F. Farahmand, and J. J. P. C. Rodrigues, "Improving vehicular delay-tolerant network performance with relay nodes," in Next Generation Internet Networks, 2009. NGI'09, 2009, pp. 1-5, http://dx.doi.org/10.1109/NGI.2009.5175762

[22] A. Jindal and K. Psounis, "Performance analysis of epidemic routing under contention," in Proceedings of the 2006 international conference on Wireless communications and mobile computing, Vancouver, British Columbia, Canada, 2006, pp. 539-544.

[23] J. Leguay, T. Friedman, and V. Conan, "Evaluating Mobility Pattern Space Routing for DTNs," in INFOCOM 2006. 25th IEEE International Conference on Computer Communications. Proceedings, 
2006, pp. 1-10, http://dx.doi.org/10.1109/INFOCOM.2006.299

[24] J. Leguay, T. Friedman, and V. Conan, "DTN routing in a mobility pattern space," in Proceedings of the 2005 ACM SIGCOMM workshop on Delay-tolerant networking, Philadelphia, Pennsylvania, USA, 2005, pp. 276-283.

[25] S. Burleigh, A. Hooke, L. Torgerson, K. Fall, V. Cerf, B. Durst, K. Scott, and H. Weiss, "Delay-tolerant networking: an approach to interplanetary Internet," Communications Magazine, IEEE, vol. 41, no. 6, pp. 128-136, 2003, http://dx.doi.org/10.1109/MCOM.2003.1204759

[26] W. Wang, T. Yang, X. Chen, X. Yao, and Q. Zhou, "Vibration energy harvesting using piezoelectric circular diaphragm array," in Applications of Ferroelectrics (ISAF/PFM), 2011 International Symposium on and 2011 International Symposium on Piezoresponse Force Microscopy and Nanoscale Phenomena in Polar Materials, 2011, pp. $1 \quad-4$, http://dx.doi.org/10.1109/ISAF.2011.6014117

[27] "Calculate Displacement as a function of Velocity, Acceleration and Time," CalculatorSoup Online Calculator Resource. [Online]. Available: http://www.calculatorsoup.com/calculators/physics/displacement_v_a_t.php. [Accessed: 30-Jun-2012].

\section{Copyright Disclaimer}

Copyright reserved by the author(s).

This article is an open-access article distributed under the terms and conditions of the Creative Commons Attribution license (http://creativecommons.org/licenses/by/3.0/). 